\title{
Agrobiodiversidad y economía de la quinua (Chenopodium quinoa) en comunidades aymaras de la cuenca del Titicaca
}

\author{
Agricultural biodiversity and economy quinoa (Chenopodium quinoa) in Aymara \\ communities of the Titicaca watershed
}

\author{
Dani E. Vargas Huanca ${ }^{1 *}$, Marti Boada ${ }^{1}$, Lenny Araca², Wilber Vargas ${ }^{3}$, Roger Vargas ${ }^{4}$
}

\section{RESUMEN}

En 2013 la FAO ha emprendido una campaña mundial, promoviendo el consumo de la quinua, la demanda internacional se incrementó rápidamente, generando complejos escenarios agroalimentarios en las comunidades aymaras de la cuenca del Titicaca "principal zona de producción de quinua a nivel mundial" con impactos en el sistema de agrobiodiversidad, en este contexto consideramos de suma importancia investigar cómo afecta la demanda internacional, la intensificación de la producción y la exportación de la quinua, al sistema de agrobiodiversidad aymara en la cuenca del lago Titicaca para lo cual previamente; (1) Determinamos los volúmenes de producción nacional y volumen de exportación desde el año 2000 a 2015 en los dos principales productores, Perú y Bolivia, analizamos su dinámica y diferencias, (2) demostramos los cambios en el sistema de agrobiodiversidad aymara con la introducción de la quinua al proceso de economía global; específicamente la tendencia de la conservación de variedades de quinua y mantenimiento de diversas especies agrícolas, se consigna además la situación de conocimientos ancestrales para el cultivo de la quinua y el uso de agroquímicos o fertilizantes sintéticos para el incremento de la productividad dentro del proceso de intensificación y la extensión del cultivo de la quinua. En conclusión, la inclusión de la quinua en la economía de libre mercado y el modelo de producción intensiva promovida por el gobierno y los agroexportadores, también legitimada por gran parte de los agricultores de la zona de estudio, la intensificación y la extensión ha reducido la cantidad de variedades de tipos de quinua y el número de especies agrícolas cultivadas en la misma zona geográfica antes del cultivo de quinua para la exportación, así como la extinción de los conocimientos ancestrales y el empobrecimiento de suelos.

Palabras clave: sustentabilidad, agrobiodiversidad, quinua, aymaras, Titicaca.

\begin{abstract}
FAO in 2013 has launched a global campaign promoting the consumption of Quinoa, international demand increased rapidly, generating complex scenarios agri Aymara communities of the Titicaca watershed "main production worldwide Quinoa" with impacts on agricultural biodiversity system, in this context we consider important to investigate how it affects international demand, the intensification of production and export of Quinoa, Aymara system of agricultural biodiversity in the Lake Titicaca for which previously; (1) We determine the volumes of domestic production and export volumes from 2000 to 2015 in two major producers Peru and Bolivia, analyze its dynamics and differences, (2) show the changes in the Aymara system with the introduction of agrobiodiversity quinoa to the process of global economy; specifically the trend of conservation varieties of quinoa and maintenance of various agricultural species, also entered the situation of ancestral knowledge for growing quinoa and use of chemicals or synthetic fertilizers to increase productivity in the process of intensification and the extent of cultivation of Quinoa. In conclusion, the inclusion of quinoa in the free market economy and the intensive production model promoted by the government and the agricultural exporters, also legitimized by the majority of farmers in the study area, the intensification and expansion has reduced the many varieties of types of quinoa and the number of agricultural crop species in the same geographical area before quinoa for export as well as the extinction of ancestral knowledge and impoverishment of soils.
\end{abstract}

Key words: sustainability, agrobiodiversity, Jirwa, Aymara, Titicaca.

\footnotetext{
Instituto de Ciencia y Tecnología Ambiental ICTA, Universidad Autónoma de Barcelona. Barcelona, España.

Universidad de Oporto. Oporto, Portugal.

3 Instituto de Investigación Interdisciplinaria IIPACH, Universidad de Salamanca. Salamanca, España.

4 Instituto de Investigación y Revaloración de Culturas Indígenas. Yunguyo, Perú.

* Autor por correspondencia: davarhua@ doctor.upv.es
}

Fecha de Recepción: 28 Abril, 2015.

Fecha de Aceptación: 31 Agosto, 2015. 


\section{Introducción}

La quinua es un grano andino con origen en la zona geográfica habitada por el pueblo aymara; el nombre en la lengua originaria es "Jirwa" y "Jupha", en el siglo XIX con la creación y el establecimiento de los Estados de Perú, Bolivia, Chile y Argentina, el territorio aymara ha sido dividido e integrado a los cuatro Estados, lo que generó impactos socioeconómicos y políticos afectando su identidad. Lo relevante de este pueblo es que mantiene aún avanzados conocimientos, etnotecnologías y saberes para un aprovechamiento ecoeficiente de la biodiversidad, producto de ello han forjado un sistema de agrobiodiversidad; miles de variedades de patata (Solanum tuberosum), miles de variedades de quinua (Chenopodium quinoa), las variedades de kañihua (Chenopodium pallidicaule), de tarwi (Lupinus mutabilis) ahora mundialmente conocidos. Según Antúnez de Mayolo, S. E. (1981) el alto valor nutricional de los alimentos andinos pronto sorprendería al mundo, décadas después la comunidad internacional en la Organización de Naciones Unidas (ONU) declararía el 2008 como "Año internacional de la patata" y cinco años después "el año internacional de la quinua 2013" y estas marcarían un hito para la agrobiodiversidad aymara que se ha venido manteniendo sostenible durante miles de años con la lógica económica aymara cosmo-biocéntrica Pachampkamaña en la cual la "tierra viva" (Reynaga, F. 1998) necesita tan igual al humano, "alimentarse, trabajar y dormir", tres reglas fundamentales de Jake (humano), de esta provienen los principios para mantener vivas las tierras agrícolas; Mankayaña (nutrir) Samartayaña (Reposar) Achokayaña (producir), el Amawta Fausto Reynaga decía "somos tierra que piensa, de ella venimos y hacia ella andamos" (Reynaga, F. 1998), las tierras agrícolas son la fuente de donde proviene el ser humano así se define dentro de la cosmovisión y racionalidad andino-amazónico, diferente de la lógica "antropocentrista" del capitalismo, este último muestra un pragmatismo funcional que considera a la tierra, la biodiversidad, el agua, como recursos capitalizables (Boada, et al. 2003) y rentables en su etapa de explotación. Según el viejo anciano aymara Apolinario "para el pueblo aymara, la labor agrícola de contacto directo con la tierra, juntamente con el descanso y la buena alimentación son considerados una celebración imprescindible para la salud físico mental humana (J. Apolinario, comunicación personal sobre "dialogo sobre la Cosmovisión Aymara y la Actividad Agrícola", en la comunidad Choquechaca, 10 de diciembre de 2013) en una lógica de economía agroalimentaria aymara basada en el autosostenimiento local, con "un alto nivel de cuidado y respeto a los ecosistemas y biodiversidad" (Murra, J.V. 1975), sin embargo con la expansión de "la modernidad y la globalización de la agroindustria solo se ha logrado que "el papel de los paisajes agrícolas en la conservación de la biodiversidad sea ignorado a pesar de su papel potencial en la conservación de especies que ahora se encuentran en declinación (Herkert, J. R., 2009), cuando en la cultura aymara se han valorado los paisajes agrícolas desde tiempos inmemoriales, lamentablemente en las últimas décadas en territorios aymaras del sur de Bolivia, "la expansión de la quinoa ya ha generado denuncias de diversos impactos negativos del cultivo de la quinoa tales como la degradación del suelo, conflictos socioeconómicos y pérdida de biodiversidad" (Winkel, et al. 2014).

Históricamente desde la cuenca del Titicaca se ha extendido su producción hacia el sur y el norte; en la actualidad comprendería los territorios ocupados por los Estados de Perú, Bolivia Chile, Argentina, Ecuador Colombia y Venezuela. Con la colonización y posterior formación de Estados modernos se fueron reduciendo las zonas de producción, pero la resistencia aymara conservó hasta estos días este sagrado alimento, cuando se descubre el alto valor nutricional que poseía esta especie y se emplea en la NASA para misiones espaciales, empieza a incrementarse la demanda y con ello la producción, principalmente en la zona aymara de Perú y Bolivia, desde 1970 aproximadamente hasta hoy, el año 2014 el 80\% de la producción peruana se ha dado en comunidades de la cuenca del lago Titicaca según datos de la FAO, MINAGRI, y PROMPERU. La globalización del comercio agroalimentario internacional ha dado espacio a mercados de alimentos para consumidores que deciden sus compras en función de su valor nutricional, ahí los productos como la quinua se han posicionado muy bien.

Los operadores del comercio internacional han proyectado estrategias inmediatas de aprovechamiento económico y nutricional de este alimento, juntamente con las instituciones públicas han venido promoviendo el cultivo de quinua a escala industrial, dejando al margen el complejo choque cosmovisionario y la interacción de racionalidades. 
Desde la institucionalidad estatal, específicamente Ministerio de Agricultura, Ministerio de Comercio Exterior, Ministerio de la Producción se dio más importancia a la atención de las propuestas de compra que se incrementaron rápidamente en Estados Unidos, Canadá, Australia, Alemania, Holanda, Japón, Brasil y otros países; esto llevó a los principales productores a nivel mundial, Perú y Bolivia, a fortalecer y promover la exportación de la quinua considerando esta como una política nacional que favorecería económicamente a las comunidades productoras. Los funcionarios públicos, los gobiernos locales, las organizaciones sociales e indígenas han extendido el mensaje de comercializar a nivel internacional mostrando principalmente los beneficios económicos que se esperarían, trabajando arduamente en: (1) la ampliación de la extensión (espacial/hectáreas) del cultivo de la quinua, (2) la intensificación de su producción por lo menos dos cosechas al año y (3) el empleo de biotecnología y de agroquímicos para alcanzar el crecimiento de la economía de la quinua en el mundo, tales lineamientos se han impulsado principalmente desde el Ministerio de Agricultura (MINAGRI) y el Instituto de Innovación Agraria (INIA). La intensificación de la producción agrícola que empezó a darse en los años 60 y 70 con la revolución verde y sus estrategias: (a) la aplicación de leyes de Mendel para la mejora de especies cultivadas, (b) el impulso de la agroquímica y la mecanización, generando un aumento espectacular de la productividad agrícola en Europa y Norteamérica, Asia y países como Argentina y México en Latinoamérica, básicamente se ha especializado en la mejora de tres cereales clave en la alimentación humana: trigo, arroz y maíz; cabe considerar como consecuencia de la sobreexplotación de tierras agrícolas, llevando al empobrecimiento de la fertilidad de los suelos en los países en vías de desarrollo (Matson, P.A., et al 1997) y (Iáñez E. 2000). Con el objetivo de mantener los niveles de crecimiento del producto bruto interno (PBI) por encima del 5\% promedio alcanzado en los últimos 10 años, las instituciones del Estado mediante la política agraria vigente y el comercio agroalimentario de libre mercado, han desplegado una fuerte campaña para la adopción de un modelo de producción intensivo y extensivo, permitiendo que los agroexportadores destinen cada vez mayor volumen al mercado internacional. En este contexto definimos como objetivo de nuestra investigación determinar las consecuencias del impacto de la economía de la quinua en el mercado global a la agrobiodiversidad aymara en las comunidades de la cuenca del lago Titicaca.

\section{Materiales y Método}

(1) Se analizaron datos cuantitativos y cualitativos de fuentes primarias existentes en las instituciones del Estado: Ministerio de Agricultura, Ministerio de la Producción, Ministerio de Comercio Exterior, Censo Nacional Agropecuario 2012. A partir de datos estadísticos de la FAO, MINAGRI y SUNAT del Perú, Viceministerio de Desarrollo Rural y Agropecuario e INEBolivia, se construye un Gráfico que indica el volumen de producción anual del 2000 al 2015 en Perú y Bolivia; (a) totalidad de producción y (b) volumen para exportación.

(2) Se encuestó a una muestra aleatoria de 200 campesinos aymaras de 20 comunidades de las provincias de Yunguyo, Chucuito y el Collao respecto del número de variedades de quinua y la cantidad de especies de la agrobiodiversidad aymara cultivados en los últimos 20 años, así como de las percepciones y preferencias frente a la demanda, sea nacional o internacional.

(3) Revisión bibliográfica de artículos científicos y tesis doctorales en relación con las variables consideradas para esta investigación, publicados y disponibles desde Scopus y Web of Science, enfocándonos para la consideración de antecedentes de la dinámica de la agricultura intensiva y extensiva en el proceso de desplazamiento a la agricultura de subsistencia/familiar/campesina en países con mayor producción agroalimentaria de cuatro continentes.

(4) Se completó dialogando con un anciano aymara de la comunidad aymara de Choquechaca de la provincia de Yunguyo-Perú.

(5) También en el proceso investigativo se ha realizado la observación experimental de la motivación y comportamiento de los productores de Jirwa (quinua) en la zona de estudio.

\section{Resultados y Discusión}

Se determina cuantitativamente la evolución del crecimiento de la producción de la quinua desde el 2000 al 2015, a partir de los datos cuantitativos obtenidos de informes anuales del Ministerio de 
Agricultura, Ministerio de la Producción, Ministerio de Comercio Exterior, Censo Nacional Agropecuario 2012, donde se observa que la producción de quinua al 2015 en los últimos 16 años se ha incrementado en más de $500 \%$, este dato concuerda con los pronósticos de la FAO, y los reportes mensuales y anuales que emite la Superintendencia Nacional de Administración Tributaria del Perú (SUNAT). En Bolivia también se ha incrementado en la misma proporción de acuerdo con las cifras obtenidas en los informes del Viceministerio de Desarrollo Rural y Agropecuario e INE-Bolivia. Con todos los datos referidos anteriormente construimos la Figura 1 que indica el volumen de producción anual en los últimos 16 años en Perú y Bolivia; (a) totalidad de producción y (b) volumen para exportación.

Tomando como premisa el primer resultado, en la encuesta directa a agricultores o campesinos aimaras se pregunta en relación con las estrategias que han tomado para aumentar el nivel de producción: los resultados nos muestran que 152 campesinos productores que representan $76 \%$ de los encuestados manifiestan que han abandonado al menos cuatro variedades de Jirwa en los últimos 20 años, 142 personas hacen referencia que en los tres últimos años han optado por cultivar solamente Janko Jirwa (quinua blanca), debido a que esta variedad es cada vez más cotizada su venta a acopiadores que destinan para la exportación: estos resultados muestran una clara disminución de la cantidad de variedades de quinua cultivadas en los 20 últimos años en las comunidades aymaras de las provincias Yunguyo, Juli, Ilave.

En la medición del impacto de la demanda y producción de la quinua hacia otras especies de la agrobiodiversidad aymara corroboramos que 85 agricultores (42\%) han dejado de cultivar al menos una de estas especies nativas: Olluco, Isaño, Tarwi, Kañiwa, para cultivar en lugar de ellos "Janko Jirwa" (quinua blanca). A la pregunta respecto de los motivos por los que han llegado a esta decisión, expresan que las prácticas y modos ancestrales de cultivo de quinua en la cual solo se permite una reducida extensión de tierras, considerándose a ello como un obstáculo para satisfacer la presión de la demanda de los acopiadores junto a autoridades locales y nacionales casi los obliga a extender el cultivo de quinua a tierras destinadas a otras especies nativas. Tales datos nos permiten determinar el significativo descenso de cantidad de especies nativas de la agrobiodiversidad aymaras cultivadas en el mismo periodo de años en las comunidades de las tres provincias Yunguyo, Juli, Ilave (Figura 2).

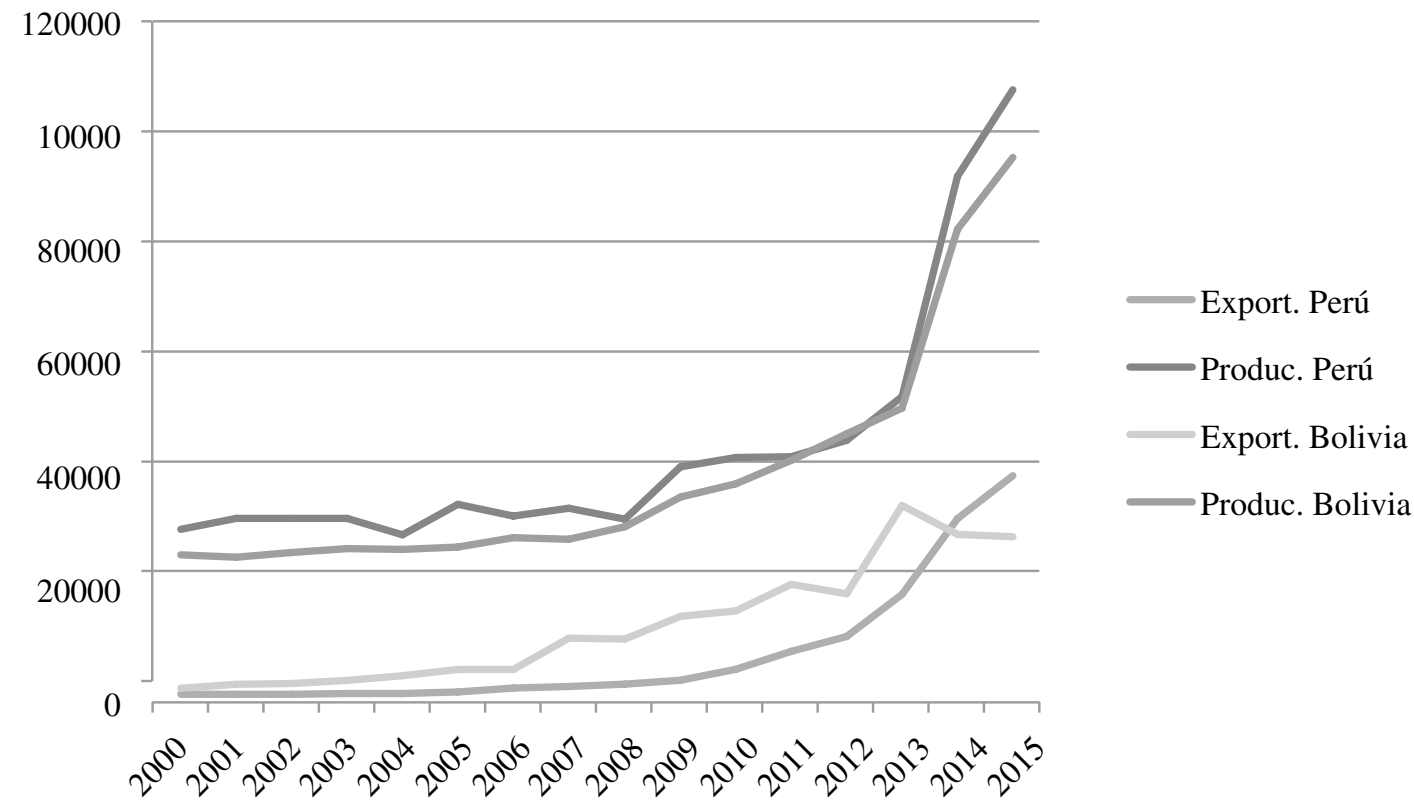

Figura 1. Volumen de producción total y de exportación Perú y Bolivia.

Fuente: Elaboración propia a partir de FAO, MINAGRI y SUNAT del Perú, Viceministerio de Desarrollo Rural y Agropecuario VDRA e INE-Bolivia. 


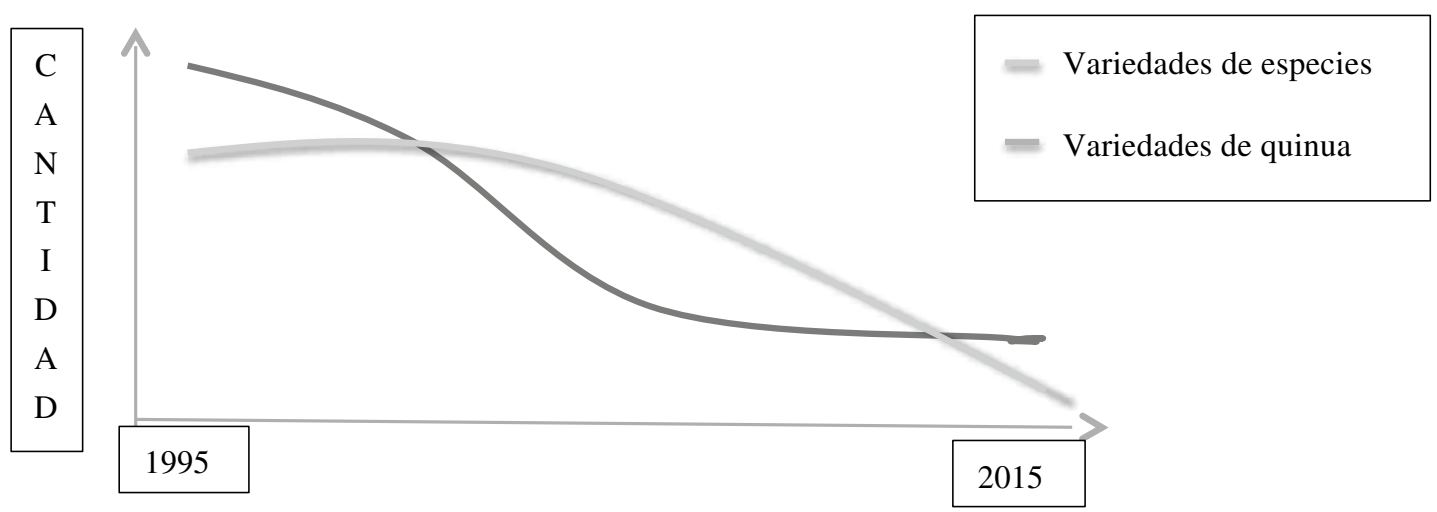

Figura 2. Dinámica de la agrobiodiversidad aymara 1995-2015.

Fuente: Elaboración propia (proyecciones elaboradas a partir de los resultados obtenidos en las encuestas).

Del diálogo con anciano Apolinario de la comunidad aymara de Choquechaca, se puede deducir el abandono de la cosmovisión y la racionalidad económica aymara por parte de los jóvenes, dejando al olvido su sistema agroalimentario, aprovechamiento del rendimiento natural o ecológico por unidad de superficie cultivada, calificada actualmente como eficiencia ecológica, lo que hacía inviable el aumento extensivo del cultivo y la producción intensiva de la quinua-Jirwa en volumen y extensión por encima de la capacidad de resiliencia de los ecosistemas. Con la caída de este sistema aymara se hace más próximo "la biotecnología europea y norteamericana con el tiempo ha desincentivado la roturación de más tierras marginales y áreas de gran valor ecológico" (Matson, P.A., et al. 1997), la implementación de un sistema de biotecnología agroalimentaria solo sería cuestión de tiempo cuando ya se han realizado investigaciones para mejoramiento genético de la quinua mediante el Instituto Nacional de Innovación Agraria, INIA; vienen implementando entre otras un banco de germoplasma de la quinua, principalmente focalizado en la región Puno mediante el Ministerio de Agricultura para la intensificación de la productividad de la quinua, esta tendencia se confirmó cuando el $40 \%$ de los productores encuestados ya tienen conocimiento de la existencia de las variedades mejoradas de quinua como el VM "INIA-Salcedo"; en la actualidad el $22 \%$ de productores cultivan esta variedad por su alta productividad económica, 163 agricultores que representan a más del $80 \%$ ya emplean agroquímicos para elevar el rendimiento productivo de sus chacras, estos resultados nos muestran la semejanza con los años 60 y 70 en la que, con la revolución verde se ha generado un aumento espectacular de la productividad agrícola en Europa y Norteamérica, Asia y países como Argentina y México en Latinoamérica, llevando hacia el monocultivo. Asimismo debemos considerar que la revisión bibliográfica nos muestra que en el Perú ya existe experiencia de un descontrolado empleo de agroquímicos, como señala SEPIA, 2007 y Herkert, J. R., 2009, "exceso de plaguicidas altamente peligrosos en la sobreexplotación de los suelos para elevar la productividad de los suelos en la región San Martín, localidad Tarapoto, donde también se señala que se viene dando una acentuada tendencia a la pérdida de biodiversidad de plantas de cultivo o también llamada erosión genética (SEPIA, 2007), en países de América Latina como Argentina, la expansión de la agricultura industrial ha dado lugar a la pérdida de las estrategias de manejo y conservación de la agricultura campesina (Cáceres, D. et al. 2010), en México, como consecuencia del impacto de las empresas transnacionales agrícolas en la agricultura local, se ve cada vez más reducida la agricultura campesina o familiar (Soto Mora, C. 1990). En India y Pakistán, con la industrialización de la agricultura, el impacto con las nuevas variedades introducidas en las prácticas agrícolas fue enorme sobre todo en su diversidad. "la biodiversidad y variedades agrícolas locales se han visto desplazadas por el monocultivo de alto rendimiento a pesar de que a menudo no rinden en las condiciones de muchas zonas tropicales o subtropicales"(E. Iáñez, 2000).

La tendencia a nivel mundial respecto de la economía agroalimentaria y la situación de la diversidad agrícola, previa consideración de nuestros 


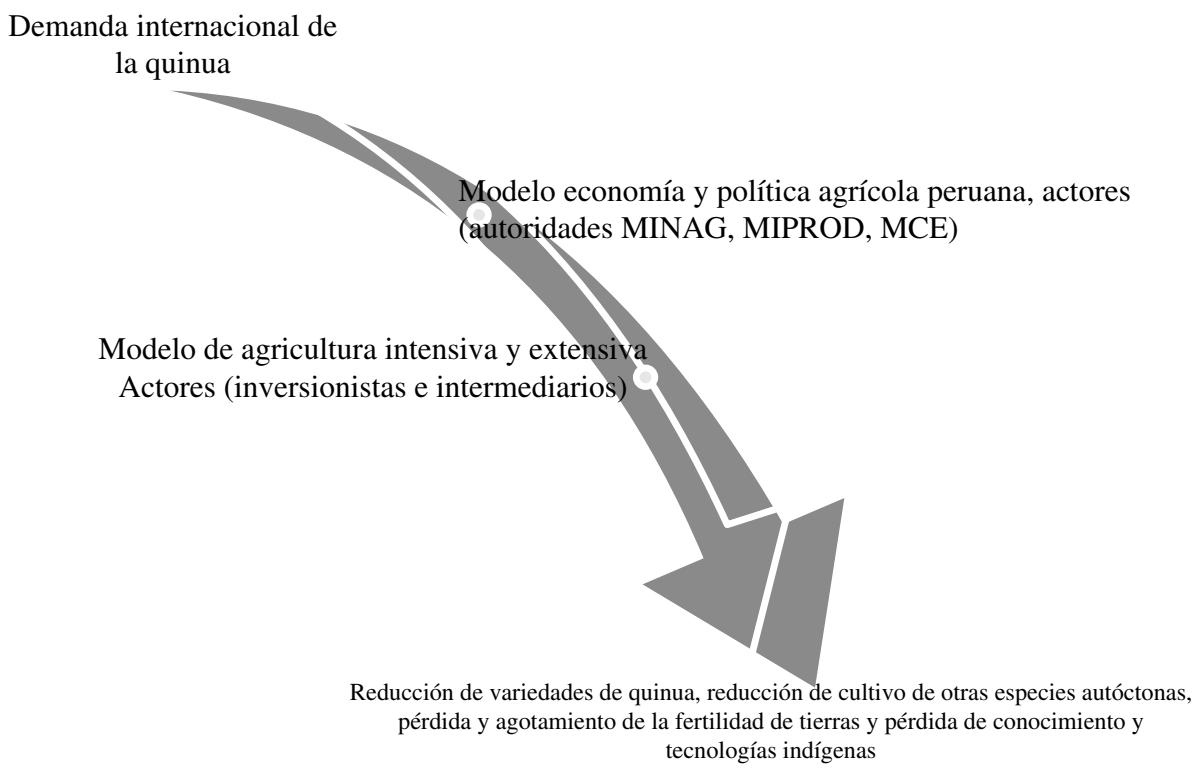

Figura 3. Relación de causa-efecto.

Fuente: Elaboración propia.

resultados así como la literatura explorada está dominada por tres factores determinantes a nivel mundial (1) la globalización, (2) la modernidad y (3) la economía neoliberal, que han traído consigo la industrialización de las actividades económicas, mediante el desarrollo tecnológico como componente principal que ha mostrado cierta eficacia en la actividad agrícola norteamericana y europea, sin embargo en los países en vías de desarrollo de los cuatro continentes restantes, las prácticas y métodos agroindustriales no han sido óptimos para el medioambiente en países como Burkina Faso, Etiopía, donde se ha profundizado la desigualdad social, ya que mediante la práctica del monocultivo "pocos alcanzaron mayores niveles de riqueza en la explotación agrícola intensiva dejando suelos degradados, mientras que la mayoría de los agricultores cada vez más pobres buscan conservar los recursos ambientales a costa del desarrollo económico y el bienestar de ajenos" (Gray, L. C., 2005).

Los agricultores usan los pesticidas disponibles incluso si estos no son apropiados para sus condiciones o condiciones de vida (Herkert, J. R., 2009), "el abuso de abonos nitrogenados y plaguicidas se intensificó en los países en vías de desarrollo, debido a que en los países avanzados el uso de fertilizantes se ha estancado, porque cantidades adicionales no redundan en mayor productividad. En África, América Latina e India en especial los países en desarrollo tuvieron que depender cada vez más de productos agroquímicos producidos por multinacionales" Enrique Iáñez (2000). Como una síntesis del análisis de los resultados se ha elaborado la (Figura 3).

\section{Conclusiones}

La producción de quinua en las dos últimas décadas, tanto en el Perú como en Bolivia al 2015 ha incrementado en cinco veces respecto del año 2000, la política agraria de corte neoliberal ha sido altamente influyente para el incremento del nivel de producción de la quinua-Jirwa, asimismo el aumento del volumen destinado para la exportación ha sido en la misma proporción, del total producido cada año desde el año 2000 hasta el 2015.

La demanda internacional de la quinua y la extensión de su producción ha causado la reducción de la cantidad de especies nativas de la agrobiodiversidad aymara cultivadas en el proceso de su evolución como Olluco, Isaño, Kañiwa, Oca, Tarwi, la economía de libre mercado y el modelo de producción intensiva promovido por los gobiernos en las dos últimas décadas, lleva a la tendencia de reducir el número de especies agrícolas cultivadas por cada familia aymara autosuficiente, con la que está llevando a una dependencia alimentaria y poniendo en riesgo su seguridad alimentaria y nutricional. 
Disminución de la cantidad de variedades de quinua funcionales al sistema de agrobiodiversidad aymara, cultivadas en los 20 últimos años en las comunidades de la cuenca debido a las experiencias mercantiles europeas y norteamericanas que al industrializar la actividad agrícola, han motivado la especialización que finalmente sería el monocultivo, básicamente al empuje de los agroexportadores, la tendencia de los productores de quinua es hacia la intensificación de la producción de Janko Jirwa, lo que genera reducción de la cantidad de variedades de tipos de Jirwa así como, por tanto es importante prever el comportamiento de la economía de Jirwa.

Respecto de los suelos; la intensificación de la producción y el abuso de agroquímicos/fertilizantes debido al desconocimiento de las consecuencias y efectos en la calidad de suelos, viene llevando a la pérdida y agotamiento de tierras fértiles, que direcciona de la misma forma a la dependencia tecnológica.

La pérdida de conocimientos y tecnologías indígenas para manejo sostenible de la economía de la quinua dentro del sistema de agrobiodiversidad aymara, se viene dando en la medida en que la tecnologías agroindustriales modernas van desplazando a la mano de obra, un fenómeno similar a experiencia de transición a la industrialización de la actividad agrícola en países como México, Argentina, (América), Burkina Faso, Etiopia (África), India, China (Asia) y España (Europa).

\section{Literatura Citada}

Antúnez de Mayolo, S. E.

1981. La Nutrición en el Antiguo Perú. Banco Central de Reserva del Perú. Lima, Perú. 189 p.

Boada, M.; Toledo, V.

2003. El planeta, nuestro cuerpo: la ecología, el ambientalismo y la crisis de la modernidad. M. Fondo de Cultura Económica. $237 \mathrm{p}$.

Chappa Santa María, C.E.; Gallusser Jacquat, S.; Polo, A.T. 2007. Perú: Sistemas productivos en la Región San Martín El problema agrario en debate. SEPIA XII, Tarapoto. Perú. $29 \mathrm{p}$.

Cáceres, D. M.; Soto, G.; Ferrer, G.; Silvetti, F.; Bisio, C. 2010. Expansion of industrial agriculture in central Argentina. its impact on peasants' strategies. Cuadernos De Desarrollo Rural, 7(64): 91-119.

Espinoza, W.

1987. Los Incas: Economía, Sociedad y Estado en la era del Tahuantinsuyo, Lima: Amaru Editores. 507 p.

Gray, L. C.

2005. What kind of intensification? agricultural practice, soil fertility and socioeconomic differentiation in rural burkina faso. Geographical Journal, 171(1): 70-82.

Herkert, J. R.

2009. Response of bird populations to farmland set-aside programs. Conservation Biology, 23(4): 1036-1040.

Iáñez, E.

2000. ¿Un papel para la biotecnología? Instituto de Biotecnología, Universidad de Granada. Disponible en: http://www.ugr.es/ eianez/Biotecnologia/agricultura.htm consultado: $21 / 03 / 15$

INEI.

2012. IV Censo Nacional Agropecuario 2012. Lima, Perú. Instituto Nacional de Estadística e Informática. 63 p.

INE-Bolivia.

2015. Información Estadística por actividad económica (agricultura). La Paz Bolivia.Disponible en: http://www. ine.gob.bo/indice/indice $\cdot$ aspx $? \mathrm{~d} 1=0201 \& \mathrm{~d} 2=6$. consultado: 21/03/15

Matson, P.A.; Parton, W.J.; Power, A.G.; Swift, M.J.

1997. Agricultural intensification and ecosystem propierties, Science, 277: 504-509.
Murra, J.

1975 Introducción. En Formaciones Económicas y Políticas del Mundo Andino, Instituto de Estudios Peruanos. Lima, Perú. pp. 19-42.

Ofstehage, A.

2012. The construction of an alternative quinoa economy: Balancing solidarity, household needs, and profit in San Agustín, Bolivia. Agriculture and Human Values, 29(4): 441-454.

PROM-Perú.

2011. Estudio de identificación de canales de comercialización y distribución de productos de Biocomercio-Granos Andinos en España.85p. Disponible en: http://www. siicex.gob.pe/siicex/documentosportal/alertas/documento/ doc/402222943rad8C13A.pdf. Consultado: 14/03/15.

Salcedo S.

2014. Comercio internacional de quinua. Capítulo 4.1. IN: Bazile D.; Bertero, D.; Nieto, C. (Eds), Estado del arte de la quinua en el mundo en 2013: FAO (Santiago de Chile) y CIRAD, (Montpellier, Francia): pp. 376-393. Disponible en: http://www.fao.org/3/a-i4042s.pdf. Consultado: 14/03/15.

Reynaga F.

1953. Tierra y libertad. La revolución nacional y el indio, La Paz, Ediciones Rumbo Sindical.

Soto Mora, C.

1990. The impact of multinational enterprises on mexican agriculture. Investigaciones Geograficas: Boletín del Instituto de Geografia, Universidad Nacional Autonoma De Mexico, 21: 57-73.

VDRA.

2015. Informe Técnico 2014 del Viceministerio de Desarrollo Rural y Agropecuario, La Paz. Bolivia. Disponible en: http:// www.agrobolivia.gob.bo/planificacion/InformeTecnico2014. pdf consultado: 14/03/15.

Winkel, T.; Álvarez-Flores, R.; Bertero, D.; Cruz, P.; del Castillo, C.; Joffre, R.; Peredo Parada, S.; Sáez Tonacca, L.

2014. Calling for a reappraisal of the impact of quinoa expansion on agricultural sustainability in the Andean highlands. Idesia, 32(4): 95-100. 
